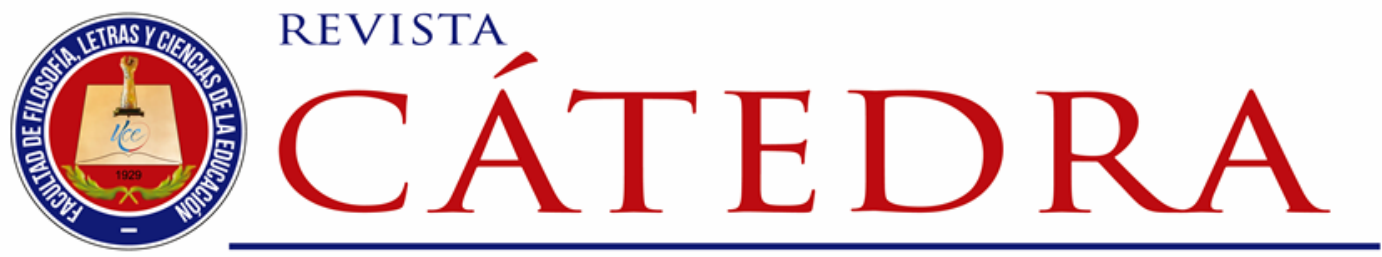

\title{
Análisis de la educación intercultural bilingüe en las instituciones educativas de la nacionalidad Tsáchila, Ecuador
}

\section{Analysis of intercultural bilingual education in educational institutions of the Tsáchila nationality}

\author{
Víctor Sánchez-Raza \\ Universidad Central del Ecuador, Quito, Ecuador \\ vasanchez@uce.edu.ec \\ https://orcid.org/0000-0003-3582-8150 \\ Magdalena Rhea-Almeida \\ Universidad Central del Ecuador, Quito, Ecuador \\ mmrhea@uce.edu.ec \\ https://orcid.org/0000-0001-7523-2664
}

(Recibido: 25/11/2019; Aceptado: 28/11/2019; Versión final recibida: 08/01/2020)

Cita del artículo: Sánchez-Raza, V. y Rhea-Almeida, M. (2020). Análisis de la educación intercultural bilingüe en las instituciones educativas de la nacionalidad Tsáchila, Ecuador. Revista Cátedra, 3(1), 46-58.

\section{Resumen}

La Educación Intercultural bilingüe (EIB) en Ecuador se ha convertido en un foco de discusión de la educación ecuatoriana debido a la convivencia de los pueblos y nacionalidades que coexisten en un mismo territorio. Uno de los principales problemas radica en el hecho de desconocer mecanismos de planeación y ejecución de políticas educativas incluyentes dado el encuentro de culturas donde la mestiza tiene prestigio y predominio sobre las demás. Por esta razón, es importante hacer visible la necesidad de la inclusión de los saberes ancestrales de la nacionalidad Tsáchila como eje transversal curricular dentro del proceso educativo intercultural bilingüe. Es necesario, entonces, evaluar la EIB, conocer cómo se expresan los saberes ancestrales en la educación e identificar las experiencias de la aplicación del Modelo del Sistema Educativo Intercultural 
Bilingüe (MOSEIB) en las instituciones educativas de las comunidades de la nacionalidad Tsáchila en el año lectivo 2018-2019. En este camino se identifican los elementos inclusivos del eje curricular transversal intercultural que deben ser fortalecidos en el Bachillerato General Unificado (BGU) para contribuir a la recuperación de la identidad cultural Tsáchila, bajo un enfoque socio-educativo, cualitativo, descriptivo y explicativo del proceso de enseñanza aprendizaje con el MOSEIB. Se han analizado sus causas, lineamientos y, recolectado información primaria y secundaria, a través de las técnicas de observación (colonos), encuesta (estudiantes), entrevista (docentes) y grupo focal (expertos). Su trascendencia radica en el aporte a la recuperación de la ancestralidad y la lucha contra la discriminación -particularmente en el ámbito educativo.

\section{Palabras clave}

Ancestralidad, discriminación, etnia, interculturalidad, MOSEIB.

\section{Abstract}

Bilingual Intercultural Education (BIE) in Ecuador has become a focus of discussion due to the coexistence of people and nationalities that coexist in the same territory. One of the main problems lies in the fact of ignoring mechanisms of planning and execution of inclusive educational policies given the encounter of cultures where the mestizo has prestige and dominance over the others. For this reason, it is important to make visible the need for the inclusion of the ancestral knowledge of the Tsáchila nationality as a curricular transversal axis in the bilingual intercultural educational process. It is necessary to evaluate the BIE, to know how ancestral knowledge is expressed in education and to identify the experiences of the Model application of the Bilingual Intercultural Educational System (MABIES) in the educational institutions of the Tsáchila nationality communities in the year 2018-2019. In this way, the inclusive elements of the cross-cultural cross-curricular axis that must be strengthened in the Unified General Baccalaureate (BGU) are identified to contribute to the recovery of the Tsáchila cultural identity, under a socio-educational, qualitative, descriptive and explanatory approach of the teaching-learning process with MABIES. Their causes, guidelines and, primary and secondary information, have been analyzed through observation techniques (settlers), survey (students), interview (teachers) and focus group (experts). Its importance lies in the contribution to the recovery of ancestrality and the fight against discrimination -particularly in the educational field.

\section{Keywords}

Ancestrality, discrimination, ethnicity, interculturality, MABIES

\section{Introducción}

La EIB se ha convertido en el foco de discusión de la educación ecuatoriana debido a la convergencia de 14 nacionalidades, con sus respectivas lenguas, en un mismo territorio. Bajo esta premisa, los gobiernos de turno no han resuelto sus limitaciones respecto a la planeación y ejecución de políticas educativas incluyentes. Esto se debe a la diversidad 
étnica y el encuentro de culturas donde el prestigio y predominio de la mestiza, es evidente. Uno de los problemas fundamentales que subyacen de este hecho es el sistema educativo cuya práctica diaria se inclina más hacia la unicidad de la educación en detrimento del desarrollo de las otras culturas que cada vez más se ven influidas por la cultura dominante.

Mantener la cultura de cada nacionalidad es un esfuerzo que sobrepasa las voluntades de las comunidades, las intenciones de sus integrantes y del mismo Estado. Y es la educación la que se ha encargado -en algunos casos- de mermar en la cosmovisión de los pueblos, sus saberes, e incluso su comportamiento social. No se puede mantener, entonces, una visión purista respecto a lo que hoy en día son las culturas, es decir, es necesario comprender que muchas viven procesos de sincretismo en ámbitos como: creencias, gastronomía, vestimenta, salud, vivienda, entre otros. Entonces, esta investigación realiza una mirada a lo que se comprende como educación intercultural aplicada a las instituciones interculturales. En ese camino, se hace una breve descripción de los orígenes de la educación en Ecuador donde se abordan temas como la Ley Orgánica de Instrucción Pública, los inicios de la Educación Intercultural Bilingüe, los Tsáchilas y la aplicación del Modelo del Sistema Educativo Intercultural Bilingüe. Luego, se explica el método que -en definitiva- es la descripción de la metodología de investigación del presente trabajo. Finalmente, se ponen de manifiesto los resultados, la discusión de resultados y las conclusiones parciales de esta investigación en curso.

\section{Origen de la educación en el Ecuador}

En el análisis del origen de la educación en el Ecuador debe tomarse en cuenta la evolución y desarrollo de las diversas culturas aborígenes -pre incásicas e incásicas- así como el desenvolvimiento de la época colonial y republicana. En primera instancia las formas de educar antes de la colonización se generaban en medio de la vida, de la convivencia comunitaria y las costumbres de sus pueblos. La oralidad y las prácticas de agricultura, caza y pesca eran las principales fuentes de aprendizaje. En la época colonial, la enseñanza se realizaba en el núcleo familiar en la mayoría de los casos; y, en pocos, en conventos donde aprendían a leer y escribir. En esta etapa se acentúan más los intereses de las clases dominantes en detrimento de los intereses de los pueblos ancestrales y de los trabajadores. El papel de la Iglesia contribuyó en el desarrollo de la educación, manteniendo una visión y misión clasista, detonándose -paralelamente- el pensamiento liberal y de ruptura con la colonia.

Más adelante, con la constitución del Estado republicano se produce la organización de la educación como un sistema. Se ampara con la institucionalización de la Dirección General de Estudios, y la Ley Orgánica de Instrucción Pública de 1906 que es una reforma a la Ley de Instrucción Pública de 1897. Esta considera entre sus postulados más importantes y enmarcada en la Revolución Liberal, la institucionalización de los colegios normales, la educación pública, laica, gratuita y obligatoria para todas las niñas y niños de cinco a doce años (LOIP, 1906. p.23). La mención de estos aspectos es importante en cuanto se desarrolla el estudio de la Pedagogía, las Ciencias Sociales, la educación superior, y con ella, el desarrollo científico y tecnológico. Como es evidente, la Educación Intercultural no puede 
quedar fuera de este proceso. En la actualidad y en un proceso histórico de extensión de la educación con las características del gobierno liberal de Alfaro, en la provincia de Santo Domingo de los Tsáchilas -y en las comunidades indígenas Tsáchilas-, se crean unidades educativas con un enfoque educativo que respeta su lengua y cultura.

\subsection{La educación intercultural bilingüe}

Parafraseando a Conejo (2008) las primeras experiencias de la EIB surgen hacia los años cuarenta en Cayambe gracias a la lidereza indígena Dolores Cacuango que, con el trabajo de un equipo de mujeres quiteñas crearon escuelas indígenas en Pichincha y, más adelante, con las misioneras lauritas las extendieron hasta Imbabura. Dejaron de funcionar hacia 1963. Por otro lado, en 1972 empiezan a funcionar las escuelas radiofónicas de los Shuar-achuar oficializándose hacia 1979. Su principal aporte es la formación de maestros indígenas. Además, aparecieron las escuelas indígenas de Simiatug en Bolívar; el Sistema de Escuelas indígenas de Cotopaxi (SEIC); la Federación de comunas Unión de Nativos de la Amazonía Ecuatoriana (FCUNAE), en 1975; el Subprograma de alfabetización kichwa que inició en 1978 con el apoyo del programa Centro de Investigaciones para la Educación Indígena (CIEI) de la Pontificia Universidad Católica del Ecuador (PUCE) y que formó un número considerable de líderes indígenas; el Chimborazoca Caipimi que fue un programa específico para la provincia de Chimborazo; el Colegio Nacional Macac, que en 1986 forma bachilleres técnicos en lengua kichwa; el Proyecto de Educación Bilingüe Intercultural, con el apoyo de Alemania; Proyecto Alternativo de educación Bilingüe (PAEBIC) de la Confederación de las Nacionalidades Indígenas de la Amazonía Ecuatoriana (CONFENIAE) y que funciona desde 1986, en ocho escuelas de Napo y Pastaza en 1986; el convenio del Ministerio de Educación y Cultura (MEC) con la Confederación de Nacionalidades Indígenas del Ecuador (CONAIE) y gracias a ello la creación de la Dirección Nacional de Educación Intercultural Bilingüe (DINEIB), en noviembre de 1988; Convenio entre el MEC y la Federación Nacional de Indígenas Evangélicos, en el año 1990 (Conejo, 2008. pp. 65-68).

El rol de los comunistas ecuatorianos en la organización indígena y campesina, levantando la aspiración de la educación intercultural bilingüe, respetando, recuperando y promoviendo su cultura, su lengua y su cosmovisión ancestral en la educación de sus pueblos, permitió el funcionamiento de las primeras Escuelas Interculturales bilingües en la serranía ecuatoriana. Este recorrido fue el antecedente para el nacimiento de lo que hoy conocemos como Educación Intercultural Bilingüe.

\subsection{Los Tsáchilas}

La nacionalidad Tsáchila, históricamente, ha logrado sobrevivir y consolidarse desde el Siglo XVIII, a través de varios hitos de reconocimiento y de inclusión desde el Estado, afirmándose en función de su idioma, su territorio, sus costumbres, su cosmovisión. No se pueden establecer fechas exactas que describan su aparecimiento. Los primeros datos que narran su existencia son los del etnólogo Paul Rivet a inicios del siglo XX.

Desde la perspectiva de Ventura (2012) la sociedad Tsáchila es un buen ejemplo del cambio de una forma de producción de auto- subsistencia a una economía orientada en parte hacia 
el mercado. Sin embargo, esta transformación no ha sido radical para ninguno de sus miembros, ni siquiera para las generaciones jóvenes que, a la vez que tratan de sacar provecho de sus tierras, siguen prefiriendo la vida en las comunidades que el trabajo asalariado en el exterior ${ }^{1}$.

Particularmente en este caso, la integración y comunalización de la sociedad Tsáchila como estrategia para proteger su territorio, para reconocer las redes de organización sociopolítica, los roles de parentesco y autoridad, hace que desde el año 1971, se reconozca la Gobernación de la nacionalidad Tsáchila. Esta gobernación es subordinada y con autonomía parcial, de acuerdo con el Instituto Nacional de Estadísticas y Censos (INEC) en el año 2010, es reconocida con 2956 habitantes (hombres 1519 y mujeres 1437), agrupados en siete comunidades: Cóngoma Grande (Santo Juan), Los Naranjos, Colorados del Búa, El Poste, Peripa, Chigüilpe, Otongo Mapalí asentados en la zona rural aproximadamente de 9060 hectáreas del cantón de Santo Domingo².

La naturaleza comunal de los Tsáchilas según Velarde ha tratado de superar el individualismo en sus formas de sobrevivencia (propia de los clanes familiares y de las contradicciones de dichos clanes), hacia las formas de labor comunitaria exclusiva de las comunidades andinas de la sierra central ecuatoriana (Velarde, 1991, p. 45-46). Sin embargo, actualmente, varios autores y entrevistados dan testimonio de un proceso no sistémico de dispersión progresiva de sus elementos identitarios culturales, producto del avance de la influencia y formas ideológicas del mestizaje ecuatoriano. El sistema de educación, incluido el sistema de educación intercultural bilingüe, no ha logrado ser el instrumento y recurso que permita canalizar la cohesión de su naturaleza cultural.

\subsection{El Modelo del Sistema de Educación Intercultural Bilingüe (MOSEIB)}

En el artículo primero de la Constitución del Ecuador (2008) se menciona que "Ecuador es un Estado constitucional de derechos y justicia, social, democrático, soberano, independiente, unitario, intercultural, plurinacional y laico. Se organiza en forma de república y se gobierna de manera descentralizada" (p. 16). Definir al Ecuador como un

1 Ventura, Montserrat (2012) Antropóloga, investigadora de la nacionalidad Tsáchila que inicia en 1991 una etnografía en territorio tsáchila (Cóngoma, Los Naranjos, Chigüilpe, Colorados del Búa y Peripa). Después de seis años de estudio, reconstruyó las relaciones sociales que se desenvuelven al interior de esta comunidad. Describió el lugar que ocupaba este grupo en la sociedad ecuatoriana, su configuración, visión del mundo, chamanismo y contribución a la destrucción del mito de las sociedades colonizadas como sociedades aculturadas.

${ }^{2}$ Contra todo pronóstico, que condenaba a los Tsáchila a la desaparición, la población ha aumentado en los últimos 30 años. Si en 1974 el MAGAP estimaba la población Tsáchila en 915 personas, en 1986 habría aumentado hasta 1403 según Robalino (1989) y el censo del Consejo Provincial de Pichincha de 1995 la acerca a 2000 personas. Las cifras con posterioridad bailan: los datos del INEC correspondientes al censo de población y vivienda realizado el 2001, establecen que los Tsáchila en Pichincha llegan a un total de 1426 aunque según el CODENPE ya en esas fechas se identifica a una minoría de 50 personas que ha migrado hacia otras regiones. Siempre según la misma fuente, el censo del 2007 indica que la población total habría aumentado de nuevo hasta 1767 y en 2010 llegaría a 2568" (Ventura, 2012, p. 44). 
Estado plurinacional es reconocer la presencia de las trece nacionalidades que interactúan en este territorio: Achuar, A'I Cofán, Huaorani, Kichwa, Secoya, Shiwiar, Shuar, Siona y Zápara en la Amazonía; Awá, Chachi, Epera y Tsáchila en la costa; y la nacionalidad Kichwa en el área andina, compuesta de diversos pueblos. Cada nacionalidad mantiene sus lenguas y culturas propias y ellas evidencian características específicas de su cosmovisión.

Muchas etnias han tenido que adaptarse a vivir en un lugar distinto del que procedían, esto por procesos históricos en los que se ha visto envuelta la sociedad. Los Tsáchilas son un ejemplo de adaptación: tratan de mantener viva su cultura y tradiciones a pesar del fuerte contacto e influencia de los mestizos de la provincia. El territorio comprende 9060 hectáreas entregadas a la nacionalidad que comprende siete comunas. La diversidad étnica constituye al Ecuador en un país culturalmente rico: gastronomía, vestimenta, cosmovisión, lengua, música, etc. Es el contacto entre las distintas etnias el que acrecienta dicha riqueza. Es por esta razón que -parafraseando a Leiva- se define a la interculturalidad como la praxis educativa intercultural, como propuesta pedagógica, democrática, participativa y antihegemónica, de justicia social que reconoce el derecho de cada estudiante a ser diferente, que rechaza cualquier forma de discriminación, que recupera los intercambios comunicativos de valores, saberes, códigos, cosmovisión entre grupos culturales distintos y en igualdad de condiciones entre los sujetos (Leiva, 2017, pp. 22-50-213).

A manera de síntesis, es necesario recuperar la idea central de los documentos de El Ministerio de Educación (2012) institución que expidió el MOSEIB, cuya misión es rescatar, conservar y desarrollar la lengua, los saberes y la cultura de los pueblos y nacionalidades indígenas. De esta manera se contribuye con la formación de seres humanos integrales, así como de la plena defensa de su territorialidad con la subsecuente revalorización de la organización social y económica de sus comunidades, mediante procesos educativos de calidad. Tanto en el sistema educativo como en la esfera social es necesario que no se restrinja la igualdad de oportunidades, respaldadas en diferencias como sexo, edad, etnia, religión, género, posición social, aptitudes. Para ello es necesario comprender que los conceptos inclusión e integración no significan lo mismo, aunque aparentan cierta similitud semántica; por otro lado, equidad e igualdad presentan la misma dificultad de definición.

En el caso de Santo Domingo de los Tsáchilas, el Ministerio de Educación creó la Dirección de Educación Intercultural Bilingüe Tsáchila (DEIBTSA), sede en Santo Domingo de los Colorados, con jurisdicción sobre todos los centros educativos comunitarios, fiscales, fiscomisionales y particulares del nivel básico, bachillerato, post bachillerato, de educación popular permanente (La Hora, 2003, sección social). Esta entidad es la encargada de asesorar, orientar y realizar el seguimiento de las acciones administrativas, financieras y técnico pedagógicas de la DEIBTSA, lo que es considerado como uno de los logros más importantes de la nacionalidad, dentro de su lineamiento, en la defensa de la educación bilingüe, lo que les debería permitir mantener la lucha por la recuperación de sus valores étnicos y culturales. 
En el análisis de la aplicación del criterio de interculturalidad, los procesos de escolarización en Ecuador no toman en cuenta las diferencias específicas de su etnia. No significa que en las leyes establecidas desde la Constitución y la Ley Orgánica de Educación Intercultural Bilingüe no se mencionen estos criterios, sino que a pesar de que se encuentran dentro de las leyes, no se las toma en cuenta en la cotidianidad. Las nuevas edificaciones construidas para fortalecer el sistema educativo se han realizado con la misma estructura sin poner de manifiesto características propias de la cultura del sector donde se las construyó. Para la Organización de las Naciones Unidas para la Educación, la Ciencia y la Cultura (UNESCO) 2005, en el Art. 4.8 de la Convención sobre la Protección y la Promoción de la Diversidad de las Expresiones Culturales, afirma que la interculturalidad "se refiere a la presencia e interacción equitativa de diversas culturas y a la posibilidad de generar expresiones culturales compartidas, a través del diálogo y del respeto mutuo" (UNESCO, 2005. Párr. 1). La implementación del MOSEIB requiere, de manera urgente, ser evaluado, particularmente después de varios años de su implementación, pues estudiosos y expertos afirman que existe un proceso de dispersión cultural que avanza día a día, producto del avance de diversas expresiones del mestizaje.

El rol del docente es trascendente en el desarrollo socioeducativo y sociocultural en las instituciones educativas de la comunidad Tsáchila, en la provincia de Santo Domingo de los Tsáchilas. Las entrevistas realizadas a expertos en la EIB de los Tsáchilas permiten concluir que los docentes desconocen las estrategias metodológicas y herramientas didácticas relacionadas al sistema educativo. Es limitado el conocimiento de la cultura y lengua tsáfiki, lo que dificulta la comprensión de su cosmovisión. Se requiere realizar un análisis del rol de la EIB en las instituciones educativas Tsáchilas con la finalidad de retroalimentar su aplicación curricular para que cumpla con sus propósitos. Caso contrario, se corre el riesgo de desacumular los iniciales esfuerzos por recuperar y fortalecer la identidad cultural de las etnias ancestrales, particularmente de la nacionalidad Tsáchila. El abordaje del análisis de este tema implica necesariamente cuestionar el sistema EIB. Este cuestionamiento incluye lineamientos y concreciones curriculares para los cuales no se ha preparado ni capacitado adecuada e integralmente al personal docente, así como del rol del gobierno central, local y de participación de la comunidad.

\section{Materiales y métodos}

Esta investigación se ha desarrollado bajo el enfoque cualitativo, pues permite la identificación de una realidad a través de una investigación comprometida con explicaciones contextualizadas. La modalidad de desarrollo de este enfoque es la de campo, documental bibliográfica y exploratoria con una perspectiva socio educativa. En primera instancia, se revisó la literatura relacionada con la nacionalidad tsáchila así como temas relacionados con la EIB. Luego, se describieron los fenómenos educativos que giran en torno a la EIB en las comunidades Tsáchilas. Finalmente, se analizan las causas y consecuencias de la aplicación de la EIB. Para la recolección de información primaria y secundaria, así como de la descripción de las variables, se han considerado las técnicas de encuesta a estudiantes; entrevista a docentes que están inmersos en el sistema MOSEIB y grupo focal a profesionales que conocen de la temática de la educación intercultural bilingüe Tsáchila. 
Los primeros datos recabados relacionados con entrevistas a autores de investigaciones similares como Ramiro Andino o Víctor Hugo Torres han sido analizados y cruzados con las definiciones teórico-conceptuales de varios autores, así como con el criterio de los investigadores. La investigación todavía está en curso.

\section{Resultados}

En las entrevistas a las fuentes de información de personas conocedoras del tema, así como de las fuentes secundarias, se han podido extraer varias afirmaciones que, correlacionadas con la operacionalización de las variables, dimensiones e indicadores, han permitido construir un criterio estructurado, tanto de la problemática como de las posibles alternativas que se podrían implementar. Entre los principales resultados extraídos de las entrevistas se encuentran los siguientes:

- "El principal problema que existe en el MOSEIB, es la escasa capacitación en educación intercultural bilingüe" (R. Andino, entrevista personal, 3 de octubre de 2019).

- Aguavil - Andino (2019) afirman "el poco incentivo de las autoridades educativas por formación de tercer nivel o capacitación con base al MOSEIB implica un estancamiento o conformismo de estos docentes por cómo imparten sus clases" ( $p$. 82).

- Aguavil - Andino (2019) al respecto de la formación docente considera que es "un punto de reflexión, incertidumbres y conflictos en todo sistema educativo; es más, en algunos casos se desestima la importancia de la formación y carrera docente para el logro de los propósitos educativos y sociales de las reformas educativas" (p. 85).

- En el bachillerato intercultural bilingüe, no se logra la concreción del diálogo de saberes, entre los componentes de la cosmovisión Tsáchila y los elementos característicos de la cultura mestiza del Ecuador. "La identidad cultural indígena Tsáchila está sufriendo un fuerte proceso de descomposición, producto del avance incesante y permanente de la mayoría mestiza" (Torres, Víctor Hugo, entrevista personal, 3 de octubre de 2019).

- De acuerdo con los resultados parciales de la presente investigación, la problemática educativa tiene entre sus principales causas la falta de formación docente y de tercer nivel de la mayoría del profesorado quienes, incluso, son docentes bachilleres. Algunos de ellos, ya no hablan en tsáfiki y, en consecuencia, no aportan al fortalecimiento de su lengua y cultura a través del sistema escolar. De esto devienen consecuencias como la falta de aplicación de nuevas metodologías de enseñanza-aprendizaje, la falta de utilización de recursos didácticos y recursos innovadores bajo una visión socio crítica de su propio entorno y, entre otras, la pérdida de su lengua ancestral.

- Otro de los elementos de la cosmovisión indígena ancestral que se vienen distorsionando, tiene relación con la territorialidad, entendida esta como la conciencia y defensa de su territorio. La educación intercultural bilingüe no desarrolla elementos que permitan afirmar esa territorialidad Tsáchila. Lo mismo 
sucede con el acoplamiento hacia las otras cultural, pues los Tsáchilas absorben mucha alienación dejando de lado su propia identidad.

- Un elemento fundamental en el análisis de esta problemática educativa es el relacionado con el origen de clase de la educación, a lo que Bruner (1991) denomina la "génesis social de los procesos cognitivos" (Rojas et al, 2011. pp. 218), cuyas formulaciones reflexivas dieron respaldo al planteamiento de Vigotsky de la educación histórico cultural. Es decir, la revolución cultural y científica tienen incidencia en las diferencias marcadas que existen en la calidad educativa en relación con los intereses de clase de quienes la dirigen, de las inequidades relacionadas con la producción social y la apropiación individual, de grupo monopólicos, de la riqueza nacional.

\section{Discusión de resultados}

Con respecto al problema del MOSEIB, en efecto, no se trata solo de la inclusión, de ese nombre, lo que genera inclusión cultural, se trata de un sistema intercultural que requiere ser contextualizado en la realidad, la naturaleza y el carácter de los Tsáchilas, así como en su lengua ancestral. No basta con solo mostrar unos pocos rótulos y/o gráficos con palabras en Tsáfiki, sino interlocutar e interactuar y, a partir de allí, consolidar la identidad y ancestralidad cultural de los Tsáchila. El estado debe formar parte, no solo de la definición del concepto "interculturalidad", sino un actor de su aplicación en todo ámbito educativo y de interacción comunicativa.

Por otro lado, la necesidad por elevar el nivel de profesionalismo de los docentes, tiene varias motivaciones que van desde la recategorización, dentro del escalafón salarial docente, pasando por el requerimiento de una interacción del proceso de enseñanza aprendizaje más calificado. Para ello hay que poner en escenario los recursos metodológicos y didácticos con el fin de recuperar las vivencias y saberes ancestrales, principalmente la lengua materna, tanto del estudiante con el docente, como entre estudiantes y entre docentes. Estas limitaciones de incentivo, pueden, de hecho, afectar el interés y motivación de los docentes que expresan frustración y desencanto por implementar su plena vocación y calidad en el desempeño laboral, soslayando su proyecto de mejora y de profesionalización docente. Este hecho afecta la misión y visión transformadora del docente, de referenciación axiológica y ética en sus estudiantes y en la comunidad. Por eso es importante partir de una primera iniciativa: valorar el trabajo del docente a través de la formación continua del profesorado.

En el ámbito curricular, el Ministerio de Educación (2017) a través del Manual de implementación de las guías de aprendizaje Integrado de educación en el MOSEIB determina que los docentes de cada institución educativa deben establecer ajustes, adaptaciones o ampliaciones a uno o varios de los componentes del Proyecto Educativo institucional (PEI) y de sus elementos curriculares, como en "el desarrollo de las destrezas y los dominios en los niños. En esta labor, el docente, debe correlacionar las destrezas y dominios con los ejes, los ámbitos de desarrollo y aprendizaje, y con los armonizadores de saberes" (p. 54), en la PCI, en el Código de Convivencia y demás. Sin embargo, las 
autoridades y docentes de las instituciones educativas interculturales bilingües aportan limitadamente en este propósito, dejando de lado e intocados los ejes curriculares interculturales transversales: cuidado del ambiente y entorno, educación para la ciudadanía, etc., que deberían ser adaptados y contextualizados en relación con la nacionalidad Tsáchila. Esto quiere decir, que el diálogo de saberes no se concreta en el microcurrículo de las IEIB y, lejos de fortalecer su propia cultura, se fortalecen los rasgos culturales de la sociedad dominante.

Puesto que en las Instituciones Educativas Interculturales Bilingües (IEIB) no existen docentes Tsáfiki hablantes, la problemática se torna compleja pues no logran desarrollar orientaciones vocacionales, razonamientos lógico matemático, lógico verbal, lógico abstracto, de Ciencias Naturales y Ciencias Sociales contextualizados con su ancestralidad y entorno propio. Resulta que lo más destacado del MOSEIB ha sido recogido de la nacionalidad Quichua. Los pocos textos en Tsáfiki son escasamente aplicados.

Uno de los resultados mencionados en el apartado anterior, tiene que ver con la distorsión de lo que se considera la defensa de la territorialidad. En realidad, no se ha comprendido qué implica tal defensa. De allí que la principal discusión que se debería plantear la comunidad y las instituciones de control y supervisión educativas, desde el Ministerio y desde el sistema MOSEIB, están relacionadas con la pregunta, “¿cómo lograr fortalecer la identidad cultural y bilingüe desde las IEIB derivados de su ancestralidad, territorialidad, saberes, identidad, etc.?". La pregunta comprende elementos que, de no intervenir e implementarse en políticas y controles de ejecución, volverán a caer en "saco roto", es decir, en la formulación de políticas -en teoría- y, con escasos y limitados resultados de aplicación -en la práctica-.

En cuanto al origen de clase en la educación, es importante enfatizar que, en la medida en que las personas cuentan con mayores ingresos económicos y facilidades materiales, lo más probable es que sus hijos lleguen a un mayor grado de escolaridad. Es un círculo vicioso, pues, en la mayoría de los casos, las personas con menor ingreso y acceso a recursos escolares no pueden acceder a un nivel de escolaridad alto. Allí, los actores de la EIB al provocar una calidad limitada de desempeño profesional generan al mismo tiempo un proceso educativo que deja sin opciones de mayor competitividad y acceso a la educación superior y a opciones laborales calificadas a los estudiantes provenientes de las nacionalidades indígenas. Las excepciones solo conforman la regla de esta realidad.

\section{Conclusiones}

Existe un proceso de dispersión cultural de los saberes ancestrales de la nacionalidad Tsáchila y, el proceso de enseñanza aprendizaje que se viene desarrollando en la educación intercultural bilingüe, no logra contener el avance del mestizaje y la dispersión cultural. Una consecuencia que deviene de este hecho es que el proceso de enseñanza-aprendizaje es poco efectivo y los conocimientos impartidos no son consecuentes con las necesidades de las comunidades Tsáchilas. 
Una de las estrategias que se están presentando y que esta investigación promueve para la recuperación de los valores y saberes ancestrales, es la conformación y funcionamiento de los denominados Centros Culturales. Ellos son espacios desde donde se practican las expresiones de la ancestralidad, territorialidad, lengua y costumbres de la nacionalidad Tsáchila. Imbricados con el turismo comunitario intercultural, requieren ser tomados en cuenta dentro de las estrategias metodológicas de la EIB, de manera tal que se logren resultados significativos en el proceso de enseñanza aprendizaje intercultural bilingüe.

La necesidad de mejoras de la formación académica y de la capacitación continua de los docentes que desempeñan su labor profesional dentro del sistema de educación intercultural bilingüe, es imperiosa. La oferta académica de las instituciones de educación superior debe adecuarse a esta necesidad. La pertinencia, entendida como la respuesta de la universidad a la solución de las expresiones de la problemática de la sociedad, debe actualizarse e incorporar opciones de procesos formativos interculturales bilingües. Esta incorporación no debe realizarse desde una visión indigenista, sino desde una visión holística e integral que tenga en cuenta la formación profesional docente con la recuperación didáctica de los saberes de la ancestralidad Tsáchilas.

La discriminación étnico-racial, de clase, género, entre otras formas de exclusión social, hace que los estudiantes que reciben una limitada educación se vean enfrentados a rupturas culturales y no logren demostrar niveles de desenvolvimiento académico (dominios, habilidades y destrezas) ni de rendimiento escolar frente al conjunto de los estudiantes mestizos. En consecuencia, la influencia de la cultura mestiza es cada vez mayor, lo que deviene en el fortalecimiento de procesos de sincretismo cultural de los Tsáchilas.

Un mejor y mayor apoyo a la investigación de procesos pedagógicos, didácticos y curriculares de la EIB, así como la producción de textos curriculares, de capacitación continua y de profesionalización docentes, serían las alternativas para enfrentar esta problemática. Lo primero es no sesgar el debate y abrirlo a todas las aristas de análisis.

\section{Agradecimientos}

Los autores expresan el agradecimiento a la Universidad Central del Ecuador que a través de sus distintos programas de capacitación ha motivado nuestras inquietudes investigativas. Expresamos nuestro reconocimiento a quienes han hecho posible el desarrollo de esta investigación, particularmente, a Ramiro Andino, Jackelline Aguavil y su grupo de profesionales de la educación con quienes nos identificamos en la experiencia de la producción académica en esta arista de la visión educativa, así como a Víctor Hugo Torres por compartir generosamente sus experiencias y valoraciones entorno a la convivencia de la nacionalidad Tsáchila y del desarrollo cultural de los habitantes de Santo Domingo.

\section{Bibliografía}

Aguavil Arévalo, J. M., - Andino Jaramillo, R. A. (2019). Necesidades formativas de docentes de Educación Intercultural Tsáchila. Alteridad, 14(1), 74-83. https://doi.org/10.17163.alt.v14n1.2019.06 
Alfaro, Eloy. (1906). Ley Orgánica de Instrucción Pública. Quito, Ecuador: Imprenta de la "Gutemberg".

Andino, R. (2019). Entrevista personal. 3 de octubre de 2019

Bruner, J. (1991). Actos de significado: más allá de la revolución cognitiva. Madrid: Alianza.

Conejo, A. (2008). Educación Intercultural Bilingüe en el Ecuador. La propuesta educativa y su proceso. Alteridad, 5(3), 64-82.

Constitución de la República del Ecuador (1998). Consejo de Desarrollo de las Nacionalidades y Pueblos del Ecuador - CODENPE

Constitución de la República del Ecuador (2008). Asamblea Nacional. Registro Oficial 449.

INEC (2010). Censo de población y vivienda 2010. Quito - Ecuador.

La Hora (2003). Se creó la Dirección de Educación Intercultural Bilingüe Tsáchila. $\begin{array}{llllll}\text { Recuperado el } & 8 & \text { de } & \text { septiembre }\end{array}$ https://lahora.com.ec/noticia/1000210866/home.

Leiva, J. (2017) La interculturalidad como respuesta preventiva del fracaso escolar en contextos de riesgo. En Anduli - Revista Andaluza de Ciencias Sociales. Málaga: Universidad de Málaga.

Ministerio de Educación (2012). Estándares de calidad educativa. Aprendizaje, Gestión Escolar, Desempeño Profesional e Infraestructura. Quito: Ministerio de Educación.

Ministerio de Educación (2017). Lineamientos pedagógicos para la implementación del Modelo del Sistema de Educación Intercultural Bilingüe. Quito: Ministerio de Educación.

Robalino, G. (1989). La verdadera gente. Una aproximación antropológica al grupo Tsachila. Quito: Consejo Provincial de Pichincha.

Rojas, L., Flórez, S., González, Y., Espíndola, L., (2011). La génesis social de los procesos cognitivos desde los planteamientos de Jerome Bruner. Prevalencia del determinismo y resurgimiento de la cultura. Tesis Psicológica, núm. 6, noviembre, 2011, pp. 215235.

Torres, J. (2019). Entrevista personal. 3 de octubre de 2019.

UNESCO (2005). La Interculturalidad. Recuperado el 9 de septiembre del 2019. http://www.unesco.org/new/es/quito/education/education-and-interculturality/.

Velarde, Patricio (1991). Santo Domingo de los Colorados y el espacio nacional (18601960). Aislamiento e integración. Quito: Ciudad. Municipio de Santo Domingo.

Ventura, M. (2012). En el cruce de caminos: identidad, cosmología y chamanismo Tsáchila. Quito FLACSO-Ecuador. Abya Yala. IFEA. Universidad Autónoma de Barcelona. 


\section{Autores}

VÍCTOR SÁNCHEZ - RAZA Magíster en Educación en la Universidad Tecnológica América en 2013. Obtuvo un curso Superior en Gestión Social y Liderazgo en la Universidad Técnica Particular de Loja auspiciado por la Fundación Esquel en 2012. Cuenta con título de Diplomado Superior en investigación Socioeducativa de la Universidad Tecnológica América en 2011. Obtuvo el título de Licenciado en Ciencias de la Educación, mención en Biología y Química por la Facultad de Filosofía, Letras y Ciencias de la Educación de la Universidad Central del Ecuador en 2007.

Actualmente es profesor titular en la Carrera de Pedagogía en Ciencias Experimentales Química y Biología de la Facultad de Filosofía, Letras y Ciencias de la Educación de la Universidad Central del Ecuador. Coordina en la Carrera la Comisión de investigación y el Proyecto Integrador de Saberes (PIS) que está en su sexta fase. Tutor de proyectos de vinculación con la sociedad desde el 2007. Tutor-director de tesis durante varios períodos académicos, así como lector y miembro de tribunales de grado. Ha realizado asesoría independiente de proyectos de investigación de varias universidades nacionales y extranjeras. Sus principales investigaciones y disertaciones se relacionan con la problemática de la educación en Ecuador en pedagogía - currículo y didáctica; de procesos andragógicos con trabajadores autónomos; de procesos interculturales e interdisciplinarios; de la realidad socio económica y política del Ecuador y el mundo. Activista por la defensa de los derechos sociales.

MAGDALENA RHEA-ALMEIDA es doctoranda de Lingüística y Lenguas en La Universidad Nacional de Rosario-Argentina cuya investigación (en proceso) se titula Proceso lingüísticos de adaptación del tsáfiki en contacto lingüístico-cultural con la sociedad dominante. Obtuvo su título de Magíster en Educación Superior por la Facultad de Filosofía, Letras y Ciencias de la Educación en la Universidad Central del Ecuador (Ecuador) en 2012 y de Licenciada en Ciencias de la Educación, mención Ciencias del Lenguaje y Literatura por la Facultad de Filosofía, Letras y Ciencias de la Educación de la Universidad Central del Ecuador en 2007.

Actualmente es profesora titular de la Facultad de Filosofía, Letras y Ciencias de la Educación de la Universidad Central Ecuador en la Carrera de Pedagogía de la Lengua y la Literatura. Es coordinadora de la Comisión de Aseguramiento de la Calidad de la misma Carrera. Catedrática y coordinadora en el área de Lingüística. Tutora-directora de tesis durante varios períodos académicos, así como lectora y miembro de tribunales de grado. Sus principales temas de investigación giran en torno a la Etnolingüística. Autora de poesía y artículos publicados en revistas independientes como Cerbatana y Periódico de Poesía (Municipio de Quito). Es parte de la obra Eros en mí de Sheyla Bravo, compiladora de las mujeres poetas ecuatorianas. Cuenta con ponencias nacionales e internacionales. 